\title{
Mechanisms of carbapenem resistance in endemic Pseudomonas aeruginosa isolates after an SPM-1 metallo- $\beta$-lactamase producing strain subsided in an intensive care unit of a teaching hospital in Brazil
}

\author{
Luciana Camila Cacci ${ }^{2}$, Stephanie Gomes Chuster ${ }^{1}$, Natacha Martins' ${ }^{2}$, \\ Pâmella Rodrigues do Carmo', Valéria Brígido de Carvalho Girão', Simone Aranha Nouér², \\ Wania Vasconcelos de Freitas², Juliana Arruda de Matos², Ana Cristina de Gouveia Magalhães², \\ Adriana Lúcia Pires Ferreira ${ }^{2}$, Renata Cristina Picão1, Beatriz Meurer Moreira' ${ }^{1 /+}$
}

'Universidade Federal do Rio de Janeiro, Centro de Ciências da Saúde, Instituto de Microbiologia, Rio de Janeiro, RJ, Brasil ${ }^{2}$ Universidade Federal do Rio de Janeiro, Faculdade de Medicina, Rio de Janeiro, RJ, Brasil

\begin{abstract}
Carbapenem-resistance mechanisms are a challenge in the treatment of Pseudomonas aeruginosa infections. We investigated changes in $\mathrm{P}$. aeruginosa carbapenem-resistance determinants over a time period of eight years after the emergence of São Paulo metallo- $\beta$-lactamase in a university hospital in Rio de Janeiro, Brazil. Patients admitted to the intensive care unit (ICU) were screened for P. aeruginosa colonisation and followed for the occurrence of infections from April 2007 to April 2008. The ICU environment was also sampled. Isolates were typed using random amplified polymorphic DNA, pulsed-field gel electrophoresis and multilocus sequence typing. Antimicrobial susceptibility was determined by disk diffusion and E-test, production of carbapenemases by a modified-CarbaNP test and presence of carbapenemase-encoding genes by polymerase chain reaction. Non-carbapenemase resistance mechanisms studied included efflux and AmpC overexpression by PABN and cloxacillin susceptibility enhancement, respectively, as well as oprD mutations. From $472 \mathrm{P}$. aeruginosa clinical isolates (93 patients) and 17 isolates from the ICU environment, high genotypic diversity and several international clones were observed; one environment isolate belonged to the $\mathrm{bla}_{S P M-1}$ P. aeruginosa epidemic genotype. Among isolates from infections, 10 (29\%) were carbapenem resistant: none produced carbapenemases, three exhibited all non-carbapenemase mechanisms studied, six presented a combination of two mechanisms, and one exclusively displayed oprD mutations. Carbapenemresistant $\mathrm{P}$. aeruginosa displayed a polyclonal profile after the SPM-1 epidemic genotype declined. This phenomenon is connected with bla $_{S P M-1}$ P. aeruginosa replaced by other carbapenem-resistant pathogens.
\end{abstract}

Key words: P. aeruginosa - SPM-1 - intensive care unit - multilocus sequence typing

The antimicrobial treatment of Pseudomonas aeruginosa infections has predominantly involved carbapenems since the 1980s (Papp-Wallace et al. 2011); then, resistance emerged worldwide (Lister et al. 2009). The mechanisms of resistance typically involve OprD porin loss, overexpression of efflux systems, overproduction of AmpC-type $\beta$-lactamase and acquisition of carbapenemase-encoding genes (Lister et al. 2009).

Although carbapenem resistance may result from several combinations of these mechanisms (RodríguezMartinez et al. 2009b, Castanheira et al. 2014), metallo$\beta$-lactamase $(\mathrm{M} \beta \mathrm{L})$ production is noted in massive outbreaks, including the international dissemination of resistant strains (Maatallah et al. 2011). Intriguingly, SPM-1 M $\beta \mathrm{L}$, which was discovered in a $P$. aeruginosa isolate in 2002, has been widely spread throughout Bra-

doi: 10.1590/0074-02760160116

Financial support: CAPES, CNPq, FAPERJ.

+ Corresponding author: bmeurer@micro.ufrj.br

Received 21 March 2016

Accepted 8 July 2016 zil (Toleman 2002); it was found elsewhere, but only after acquisition from Brazil (Cornaglia et al. 2011). Some bla ${ }_{\mathrm{SPM}-1}$-carrying isolates also produce RmtD, which confers aminoglycoside resistance by $16 \mathrm{~S}$ rRNA methylation (Doi et al. 2007). P. aeruginosa carrying bla $a_{\mathrm{SPM}-1}$ and rmtD1 have potentially become widespread in Brazilian environments, as suggested by a strain isolated from a river crossing in the city of São Paulo (Fontes et al. 2011). The $b l a_{\mathrm{SPM}-1}$ gene is mostly related to a pulsed-field gel electrophoresis (PFGE) type of ST277 (Silva et al. 2011).

SPM-1-producing $P$. aeruginosa clinical isolates that are exclusively susceptible to colistin were identified among patients admitted to a university hospital in Rio de Janeiro, Brazil, from 1999 to 2000 (Pellegrino et al. 2002). In the present study, we aimed to compare the resistance levels of $P$. aeruginosa clinical isolates detected from 2007 to 2008 with those previously described (Pellegrino et al. 2002) and to characterise the mechanisms of carbapenem resistance.

\section{MATERIALS AND METHODS}

Study design and intensive care unit (ICU) environment screening - We conducted the present study at a teaching hospital in the city of Rio de Janeiro, Brazil, from April 14, 2007 through April 14, 2008. Patients admitted to the ICU for at least $72 \mathrm{~h}$ were included in a 
prospective cohort study following patients from admission to discharge. Screening for $P$. aeruginosa colonisation in respiratory secretions and rectal swab specimens was performed at admission, on the third day of hospitalisation and then weekly until discharge. The infection control committee defined patients with infections according to established criteria (Horan et al. 2008). The hospital's Institutional Review Board approved the study (protocol No. 120/06).

Specimens from the hands of the ICU healthcare staff, sinks, faucets, benches, bed rails, mattresses, top of tables and haemodialysis portable equipment $(n=29)$ were screened for $P$. aeruginosa isolates. These specimens were collected at two time points: April 1 and April 8,2008 . Handprints from health care workers were made on blood agar. Environmental surfaces were screened with moistened swabs, inoculated onto MacConkey broth (Difco Laboratories, Detroit, MI, USA), incubated at $35^{\circ} \mathrm{C}$ overnight and identified as described below.

Patient population and P. aeruginosa isolates - A total of 502 patients were admitted to the ICU; 235 (47\%) stayed in hospital for at least $72 \mathrm{~h}$ and were eligible for the study. One patient did not agree to participate; therefore, 234 patients were included in the study, as detailed in Fig. 1. P. aeruginosa episodes of infections $(\mathrm{n}=35)$ were detected in $30(13 \%)$ of the patients. The most common was bloodstream infection $(\mathrm{n}=21)$, and many of these cases $(\mathrm{n}=11)$ were secondary to ventilator associated pneumonia (VAP, $\mathrm{n}=8)$ and intra-abdominal infection $(\mathrm{n}=3)$. Other primary infections included VAP $(n=8)$, vasculitis $(\mathrm{n}=3)$, and tracheal, intra-abdominal, and urinary tract infection (one each). A collection of 465 P. aeruginosa isolates was recovered from patients and saved, and 17 isolates were obtained from ICU environment and saved.

Bacterial identification - Clinical and environmental isolates were grown on MacConkey agar and incubated at $35^{\circ} \mathrm{C}$ for $48 \mathrm{~h}$. Blood samples were inoculated into
BacT/ALERT (BioMerieux, Askim, Sweden) bottles and subcultured onto blood agar and chocolate agar. Gramnegative bacilli, oxidase-positive, non-fermenters, and motile isolates and isolates capable of arginine decarboxylation were presumptively classified as $P$. aeruginosa. Identification was confirmed by MALDI-TOF as previously described (Bizzini et al. 2010). All isolates exhibited a score value greater than 2.0, which guarantees a reliable species identification. Isolates were stored as suspensions in a $10 \%(\mathrm{wt} / \mathrm{vol})$ skim milk solution containing $10 \%$ (vol/ $/ \mathrm{vol})$ glycerol at $-20^{\circ} \mathrm{C}$.

Antimicrobial susceptibility - Susceptibility tests with the disc-diffusion method were performed as recommended by Clinical and Laboratory Standards Institute (CLSI 2013) for the following antimicrobial agents: amikacin, cefepime, ceftazidime, ciprofloxacin, gentamicin, imipenem, meropenem, piperacillin/tazobactam and tobramycin. Colistin minimum inhibitory concentrations (MIC) were determined by broth microdilution (CLSI 2013). To calculate the resistance percentages, a single isolate was selected per patient. When several isolates were available from the same patient, we selected the isolate resistant to the greatest number of drugs. Resistance percentages were compared with those obtained in a previous study (Pellegrino et al. 2002).

Carbapenemase production and carbapenemaseencoding genes - All ceftazidime or imipenem resistant isolates were submitted to the $\mathrm{M} \beta \mathrm{L}$ double-disc synergy test, as previously described (Picão et al. 2008). Isolates from infections were analysed by a modified CarbaNP test (Nordmann et al. 2012). In brief, cell lysis was performed by 30 -min bath sonication instead of using commercial lysis buffer, and imipenem-cilastatin was used as substrate. Isolates exhibiting a positive $\mathrm{M} \beta \mathrm{L}$ double-disc synergy test or modified CarbaNP test were screened for carbapenemase encoding genes ( $b l a_{\mathrm{IMP}-\mathrm{type}}, b l a_{\mathrm{VIM-type}}$, $b l a_{\mathrm{SPM}-1,}, b l a_{\mathrm{SIM}-1,}, b l a_{\mathrm{GIM}-1,}, b l a_{\mathrm{NDM}-1}, b l a_{\mathrm{KPC}}$ and $\left.b l a_{\mathrm{GES}-\mathrm{like}}\right)$

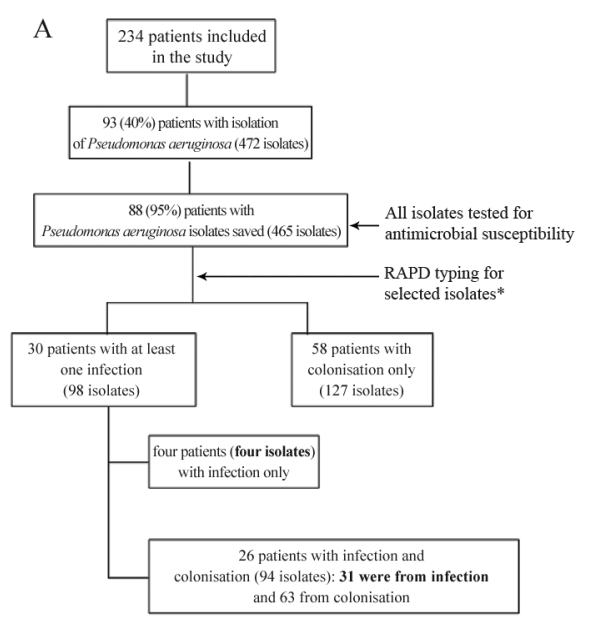

B

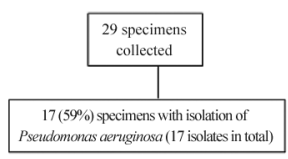

Fig. 1: patient population and bacterial isolates studied. Clinical isolates are shown in A; environmental isolates are shown in B. Isolates marked in bold were selected for analysis of mechanism of carbapenem resistance. *Isolates selected for random amplified polymorphic DNA: all from infection and a sample from colonisation, as described in methods. 
by polymerase chain reaction (PCR) (Poirel et al. 2000, Hossain et al. 2004, Mendes et al. 2007). Distinction of the $b l a_{\mathrm{GES}}$ type was achieved by gene sequencing.

Detection of non-carbapenemase resistance mechanisms - Mechanisms of carbapenem resistance other than carbapenemase production were investigated in carbapenem-resistant $(\mathrm{n}=10)$ and carbapenem-susceptible $(\mathrm{n}=25)$ isolates from infection episodes, including oprD gene mutations, overexpression of efflux systems and overproduction of AmpC-type $\beta$-lactamase.

The entire oprD gene (1412 bp) was sequenced as previously described (Rodríguez-Martinez et al. 2009b). OprD deduced amino acid sequences were compared with $P$. $a e$ ruginosa PAO1 (GenBank AAG04347.1) using Bioedit (v. 7.0.9.0) to investigate structural modifications (Hall 1999).

The MIC of imipenem and meropenem were determined by Etest (AB Biodisc, Solna, Sweden) and were compared with results obtained after incorporation of the efflux system inhibitor PA $\beta \mathrm{N}(50 \mu \mathrm{M})$ (Lomovskaya et al. 2001) and the AmpC inhibitor cloxacillin $(250 \mathrm{mg} / \mathrm{L})$ (Rodríguez-Martinez et al. 2009a) to the medium. In this comparison, at least two-fold dilution differences indicated efflux system overexpression or AmpC-type $\beta$-lactamase overproduction (Rodríguez-Martinez et al. 2009a).

Detection of RmtD 16S rRNA methylase encoding gene - Amplification of rmtD gene was performed as described (Corrêa et al. 2014) for the isolates from infection $(\mathrm{n}=35)$ of the present study and the previously characterised aminoglycoside resistant isolates $(n=15)$ (Pellegrino et al. 2002).

Strain typing - Typing by randomly amplified polymorphic DNA PCR (RAPD-PCR) (Mahenthiralingam et al. 1996) was performed with the aim of screening for main clusters of types during the study. For the RAPD test, a subgroup of isolates from clinical infections and a sample from colonisations, including at least one isolate obtained during each week of admission per patient, were selected. When multiple isolates were available, we chose the one resistant to the higher number of antimicrobial agents. In addition, we performed RAPD typing for all 17 environmental isolates. DNA band patterns were analysed with Gel Compar software version 4.01 (Applied Maths, Kortrijk, Belgium), and isolates with $100 \%$ similarity were included in a single RAPD-type.

PFGE was performed for a smaller collection of isolates as a more robust and discriminatory method as preselection for multilocus sequence typing (MLST). The PFGE sub-group included isolates from infection and isolates from colonisation obtained immediately before the onset of infection. PFGE was performed as described (Pellegrino et al. 2002). Briefly, DNA plugs were digested with SpeI (10 U/isolate, Promega, Madison, US) at $37^{\circ} \mathrm{C}$; band patterns were analysed by Gel Compar and interpreted by visual inspection. Isolates with up to two band differences corresponding to $90 \%$ similarity were included in a single pulsotype.

For MLST, the selection included 11 clinical isolates from the predominant pulsotypes and 4 ICU environment isolates (one highly resistant and one susceptible isolate of a dominant RAPD-type, the single RAPD-type also found in a patient, and the $b l a_{\mathrm{SPM}-1}$ carrying isolate). The MLST scheme available at University Warwick, UK, was performed with modifications (Curran et al. 2004). Adjustments were necessary due to several non-specific amplifications with primers proposed by these authors; thus, sequencing was not feasible. New primers were designed with primer-blast assistance (http://www.ncbi.nlm.nih. gov/tools/primer-blast) and used for both amplification and sequencing, with exception of $\operatorname{aroE}$ (Table I). A fresh bacterial suspension in water showing turbidity equivalent to MacFarland's scale 0.5 was used as template DNA. PCR parameters were initial DNA denaturation at $96^{\circ} \mathrm{C}$ for $10 \mathrm{~min} ; 35$ cycles of $96^{\circ} \mathrm{C}$ for $1 \mathrm{~min}$, the appropriate annealing temperature (Table I) for $30 \mathrm{~s}$, and $72^{\circ} \mathrm{C}$ for 1 $\mathrm{min}$; and a final extension step at $72^{\circ} \mathrm{C}$ for $7 \mathrm{~min}$. PCR products were purified by ExoSAP-IT (Affymetrix, Inc., Santa Clara, CA) and submitted to direct sequencing at the DNA Sequencing Facility of Macrogen Korea (Geumcheoun-gu, Seoul, Korea). MLST clonal complexes (CC) were defined as ST with five or more identical alleles by eBURST analysis with PHYLOVIZ version 1.0 (Francisco et al. 2012). A previously characterised isolate was also typed: isolate PHU169 (bla SPM-1 $_{1}$ positive and widespread among four hospitals in Rio de Janeiro, of RAPD-type "a" and PFGE-type "A") (Pellegrino et al. 2002).

Statistical analysis - Differences between the proportions were compared using Chi-square or Fisher's exact tests. Differences were considered significant at p-values $<0.05$.

\section{RESULTS}

Antimicrobial susceptibility, carbapenemase production and carbapenemase-encoding genes - We tested all 465 isolates recovered for susceptibility to amikacin, cefepime, ceftazidime, ciprofloxacin, colistin, gentamicin, imipenem, meropenem, piperacillin-tazobactam and tobramycin. Table II presents the comparison of the non-susceptible isolates from the present study and those previously obtained (Pellegrino et al. 2002). The results for isolates of the present study were additionally interpreted using CLSI (NCCLS) 1999 breakpoints. Amikacin resistance was significantly reduced in the current study isolates $(\mathrm{p}=0.01)$. ICU environment isolates included seven (41\%) that were susceptible to all antimicrobials and a few resistant to other agents; the ciprofloxacin resistance rate $(53 \%)$ was the highest. In total, 79 clinical and five environment isolates were resistant to ceftazidime or imipenem and were submitted to $\mathrm{M} \beta \mathrm{L}$ production double-disc synergy test. A single environment isolate (CTI33) recovered from haemodialysis portable equipment had a positive $\mathrm{M} \beta \mathrm{L}$ result.

We performed PCR for carbapenemase-encoding genes and the CarbaNP test in the sub-group of isolates including the 35 isolates from clinical infections (observed in 30 patients) and CTI33 (isolate from haemodialysis portable equipment). CTI33 was the single isolate carrying a carbapenemase-encoding gene, $b l a_{\mathrm{SPM}-1}$, that exhibited a positive CarbaNP test. Four clinical isolates carried $b l a_{\text {GES-1 }}$, a $\beta$-lactamase without carbapenemase activity. 
TABLE I

Oligonucleotides used for multilocus sequence typing

\begin{tabular}{|c|c|c|c|c|c|c|}
\hline Gene target & Primer name & $\begin{array}{l}\text { Sequence } \\
\left(5^{\prime} \text { to } 3 \text { ') }\right.\end{array}$ & $\begin{array}{l}\text { Amplicon size } \\
\text { (bp) }\end{array}$ & Nt location & $\begin{array}{l}\mathrm{AT} \\
\left({ }^{\circ} \mathrm{C}\right)\end{array}$ & Reference \\
\hline \multirow[t]{2}{*}{$\operatorname{acs} \mathrm{A}$} & $\operatorname{acs} A-F$ & GCCACACCTACATCGTCTAT & 520 & $935-1454 b p$ & 53 & Curran et al. (2004) \\
\hline & acsA-R & ACGAAGCGGTCATGGTC & & & & Present work \\
\hline \multirow{2}{*}{$\operatorname{aroE}, \mathrm{amp}$} & aroE-F & CACTCCGGCAAAGGAACGA & 867 & $127-993 b p$ & 55 & Present work \\
\hline & aroE-R & CTCAAATGCCGCCTGACAAC & & & & Present work \\
\hline \multirow[t]{2}{*}{$\operatorname{aroE}$, seq } & aroE-F & TGATCCACCGCCTGTTCG & 676 & $53-728 b p$ & 50 & Present work \\
\hline & aroE-R & ACCAGCATGCCCAGGC & & & & Present work \\
\hline \multirow[t]{2}{*}{ guaA } & guaA-F & CGACAAGGTCACCGAGATGC & 652 & 432-1083bp & 57 & Present work \\
\hline & guaA-R & GACGTTGTGGTGCGACTTGA & & & & Present work \\
\hline \multirow[t]{2}{*}{ mutL } & mutL-F & GCGACCTGTTCTTCAACAC & 530 & $455-984 b p$ & 53 & Present work \\
\hline & mutL-R & GGTGCCATAGAGGAAGTCAT & & & & Present work \\
\hline \multirow[t]{2}{*}{ nuoD } & nuoD-F & TCGATCCCTACTCCCTGTCC & 600 & $521-1120 \mathrm{bp}$ & 53 & Present work \\
\hline & nuoD-R & CCAGCTTGTCCCAGCC & & & & Present work \\
\hline \multirow[t]{2}{*}{ ppsA } & ppsA-F & GGCCAAGCAGGCCAT & 578 & 891-1468bp & 50 & Present work \\
\hline & ppsA-R & GRTTGCCGACGTTCATCAT & & & & Present work \\
\hline \multirow[t]{2}{*}{$\operatorname{trpE}$} & $\operatorname{trpE-F}$ & ATCAAGGACTACATCCTGGC & 727 & 697-1423bp & 55 & Present work \\
\hline & trpE-R & TGATGGTTTCTTCCCACTCC & & & & Present work \\
\hline
\end{tabular}

AT: annealing temperature; bp: base pairs; amp: amplification; seq: sequencing; Nt: nucleotide, referring to numbers of gene sequence of PAO1 (Genebank NC_002516.2).

TABLE II

Antimicrobial resistance rates among Pseudomona aeruginosa isolates of the present study and those previously described (Pellegrino et al. 2002)

\begin{tabular}{|c|c|c|c|}
\hline \multirow[b]{3}{*}{ Antimicrobial agent } & \multicolumn{3}{|c|}{ Number and (\%) of resistant isolates } \\
\hline & \multicolumn{2}{|c|}{ Clinical isolates } & \multirow{2}{*}{$\begin{array}{l}\text { Environmental isolates } \\
\text { from present study } \\
\qquad \begin{array}{c}(2007-2008) \\
n=17\end{array}\end{array}$} \\
\hline & $\begin{array}{c}\text { Present study } \\
(2007-2008) \\
n=88^{a}\end{array}$ & $\begin{array}{l}\text { Pellegrino et al. (2002) } \\
\qquad \begin{array}{c}(1999-2000) \\
n=115^{b}\end{array}\end{array}$ & \\
\hline Amikacin & $16(18.2)$ & $41(35.6)^{*}$ & $5(29.4)$ \\
\hline Cefepime & $33(37.5)$ & $47(41.0)$ & $3(17.6)$ \\
\hline Ceftazidime & $33(37.5)$ & $42(36.5)$ & $5(29.4)$ \\
\hline Ciprofloxacin & $30(34.1)$ & $49(43.0)$ & $9(52.9)$ \\
\hline Gentamicin & $30(34.1)$ & $55(48.0)$ & $7(41.2)$ \\
\hline Imipenem & $31(35.2)-27(30.7)^{c}$ & $44(38.3)$ & $4(23.5)$ \\
\hline Meropenem & $24(27.3)-20(22.7)^{\mathrm{c}}$ & $35(30.4)$ & $1(5.9)$ \\
\hline Piperacillin-tazobactam & $32(36.4)-25(28.4)^{c}$ & $42(36.5)$ & 0 \\
\hline Tobramycin & $29(33.0)$ & Not tested & $8(47.0)$ \\
\hline
\end{tabular}

One isolate with resistance to the greatest number of antimicrobial agents was selected per patient from a total of $225^{\mathrm{a}}$ or $200^{\mathrm{b}}$; all isolates were susceptible to colistin (minimum inhibitory concentration $<2 \mu \mathrm{g} / \mathrm{mL}$ ). Data for interpretation with Clinical and Laboratory Standards Institute (NCCLS) 1999 are presented in ${ }^{c}$; *: comparison between clinical isolates exhibited $\mathrm{p}=0.01$; other comparisons were p-values $>0.06$.

Detection of non-carbapenemase resistance mechanisms - The 35 isolates from clinical infections, including $10(29 \%)$ and $7(20 \%)$ that exhibited imipenem and meropenem resistance, respectively, were selected for determination of carbapenem-resistance mechanisms.
Efflux overexpression, AmpC-type $\beta$-lactamase overproduction and oprD gene mutations were frequently observed, as described in Table III. Sequences of the oprD gene from imipenem-susceptible isolates were similar to that of the $P$. aeruginosa PAO1 strain. In ad- 
TABLE III

Resistance marker present in 35 Pseudomonas aeruginosa isolates according to carbapenem susceptibility

\begin{tabular}{|c|c|c|c|c|c|c|}
\hline \multirow[b]{3}{*}{ Resistance mechanism } & \multicolumn{6}{|c|}{ Number and (\%) isolates } \\
\hline & \multicolumn{3}{|c|}{ Imipenem } & \multicolumn{3}{|c|}{ Meropenem } \\
\hline & R (10) & $\mathrm{S}(25)$ & $\mathrm{p}$ & $\mathrm{R}(7)$ & $\mathrm{S}(28)$ & $\mathrm{p}$ \\
\hline Efflux system & $6(60)$ & $1(4)$ & 0.001 & $4(57)$ & $3(11)$ & 0.03 \\
\hline AmpC & $6(60)$ & $4(16)$ & 0.03 & $4(57)$ & $6(21)$ & 0.16 \\
\hline oprD mutation & $10(100)$ & $0(0)$ & $<0.001$ & $7(100)$ & $3(11)$ & $<0.001$ \\
\hline
\end{tabular}

R: resistant; S: susceptible.

TABLE IV

Mechanisms of carbapenem resistance and antimicrobial resistance among clinical Pseudomonas aeruginosa isolates from infection

\begin{tabular}{|c|c|c|c|}
\hline \multirow{2}{*}{$\begin{array}{l}\text { Mechanisms of resistance: } \\
\text { efflux system, AmpC, } \\
\text { oprD mutation* (number of isolates) }\end{array}$} & \multirow{2}{*}{$\begin{array}{l}\text { Antimicrobial resistance profile** } \\
\text { (number of isolates) }\end{array}$} & \multicolumn{2}{|c|}{$\begin{array}{l}\mathrm{MIC} * * * \\
(\mu \mathrm{g} / \mathrm{mL})\end{array}$} \\
\hline & & IPM & MER \\
\hline \multirow[t]{3}{*}{,,$+++(3)$} & IPM MEM FEP CIP CAZ TZP TOB GEN (1) & 32 & 32 \\
\hline & IPM MEM (1) & 24 & 12 \\
\hline & IPM (1) & 12 & 4 \\
\hline \multirow[t]{3}{*}{,,$+-+(3)$} & IPM MEM AMK FEP CIP CAZ TZP TOB GEN (1) & 24 & $>32$ \\
\hline & IPM MEM FEP CIP CAZ TZP TOB GEN (1) & 16 & $>32$ \\
\hline & IPM (1) & 6 & 1.5 \\
\hline \multirow[t]{3}{*}{,,$-++(3)$} & IPM MEM FEP CIP CAZ TZP TOB GEN (2) & 24 & $>32$ \\
\hline & & 32 & $>32$ \\
\hline & $\operatorname{IPM}(1)$ & 16 & 2 \\
\hline,,$--+(1)$ & IPM MEM FEP CIP CAZ TZP TOB GEN (1) & 4 & 6 \\
\hline,,$++-(1)$ & Susceptible (1) & 1.5 & 0.38 \\
\hline,,$-+-(3)$ & Susceptible (3) & $1.5-0.75$ & $0.19-0.38$ \\
\hline \multirow[t]{5}{*}{,,$---(21)$} & AMK FEP CIP CAZ TZP TOB GEN (2) & $0.75-1.5$ & $0.094-2$ \\
\hline & AMK FEP CIP CAZ TOB GEN (2) & 0.75 & 1 \\
\hline & AMK FEP CAZ PIP (1) & 0.75 & 0.19 \\
\hline & TOB, GEN (1) & 1 & 0.25 \\
\hline & Susceptible (15) & $0.5-1$ & $0.064-0.5$ \\
\hline
\end{tabular}

*: assessed by comparison of carbapenem minimum inhibitory concentration (MIC) and in combination with PA $\beta \mathrm{N}$ or cloxacillin and sequencing of $\operatorname{pr} D$. Antimicrobial susceptibility was determined by disc diffusion (**) or E-test (***); + or - : presence or absence of mechanism of resistance, respectively; AMK: amikacin; CAZ: ceftazidime; CIP: ciprofloxacin; FEP: cefepime; GEN: gentamicin; IPM: imipenem; MEM: meropenem; TZP: piperacillin-tazobactam; TOB: tobramycin; IMP and MER MIC interpretation: $\leq 2$, susceptible; 4 , intermediate; $\geq 8$, resistant.

dition, imipenem-resistant isolates had oprD gene inactivating mutations: a frameshift produced by deletions $(\mathrm{n}=6 ; 60 \%)$ resulting in premature stop codons was the most frequent, followed by base pair substitutions $(n=3$; $30 \%)$ and a 4-bp insertion $(n=1 ; 10 \%)$ in the coding sequence. Imipenem resistance was statistically associated with efflux overexpression and AmpC-type $\beta$-lactamase overproduction $(\mathrm{p}<0.05)$ and completely matched oprD mutation. Meropenem resistance was statistically asso- ciated with efflux overexpression and oprD mutation ( $\mathrm{p}$ $<0.05)$. AmpC-type $\beta$-lactamase overproduction was increased (57\%) among resistant isolates compared with susceptible isolates $(21 \%)$, but the difference was not significant $(\mathrm{p}=0.1)$. The combinations of these carbapenem resistance mechanisms are presented in Table IV.

Detection of RmtD $16 S$ rRNA methylase encoding gene - The rmtD gene was not detected among isolates from infections, whereas seven $(47 \%)$ of 15 clinical iso- 
lates of the previous study (Pellegrino et al. 2002) carried this gene. Five of these seven were $b l a_{\mathrm{SPM}-1}$ positive, including isolate PHU169.

Strain typing - A diverse clonal population was observed among the subgroup of 225 clinical isolates and was selected for typing (according to the criteria described in methods). A total of 106 (47\%) isolates were included in 34 RAPD-types with clusters (each with 2-10 isolates), and 119 were unique genotypes. One clinical isolate was not typeable. Fig. 2 highlights the temporal distribution of RAPD types detected in at least four clinical isolates. The 17 ICU environment isolates were clustered in 10 RAPD types. One genotype recovered from a sink drain was indistinguishable from two clinical isolates; in addition, the RAPD-type of CTI33 was indistinguishable from that of the $b l a_{\mathrm{SPM}-1}$ positive control isolate (PHU169).

A total of 33 isolates from infection, 22 isolates from colonisation and the ICU environment isolate CTI33 were typed using PFGE (two isolates from infection were PFGE-untypeable). Isolates were included in 37 PFGE-types: two with six isolates each, two with three isolates each, nine with two isolates each, and 24 were unique. Only the CTI33 had a band profile indistinguishable from the SPM-producing control isolate PHU169.

Isolate CTI33 and control PHU169 were the only ones included in ST277 (Table V). No clinical isolate belonged to this clone. Three new allele combinations were detected (ST1602, ST1603 and ST1844) for clinical and environmental isolates.

\section{DISCUSSION}

The present study aimed to investigate the fate of $P$. aeruginosa that is resistant to carbapenems due to SPM-1 production after its previously reported explosive emergence at a university hospital in Rio de Janeiro, Brazil (Pellegrino et al. 2002). A cohort of patients was thoroughly screened for $P$. aeruginosa infection and monitored for one year. No strain with an SPM-1 compatible phenotype was identified among 465 clinical isolates, as revealed by a negative $\mathrm{M} \beta \mathrm{L}$ double-disc

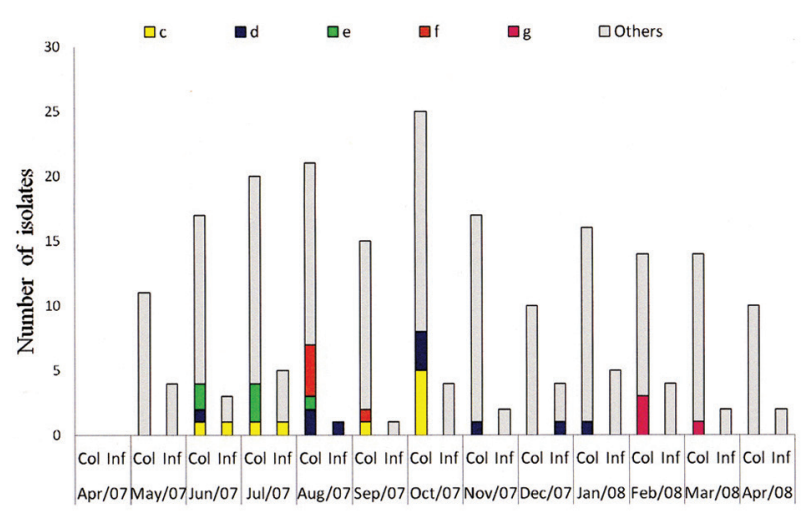

Fig. 2: temporal distribution of random amplified polymorphic DNA-types among Pseudomonas aeruginosa isolates from colonisation and infection. synergy test for all isolates exhibiting ceftazidime or imipenem resistance. It is still possible that the $b l a_{\mathrm{SPM}-1}$ gene was present in a carbapenem- or ceftazidime-susceptible isolate; however, this resistance determinant has been rarely described in carbapenem-susceptible isolates (Pellegrino et al. 2008, Picão et al. 2012). We obtained a single $b l a_{\mathrm{SPM}-1}$ carrying isolate from the environment. Apparently, SPM-1-producing isolates of the pandemic lineage ST277 were replaced by polyclonal $P$. aeruginosa. The temporal distribution of $P$. aeruginosa isolation and the predominant RAPD types revealed an endemic fluctuation of cases with no major clusters or types in the study. The subsequent scenario followed the globalisation trend and included the eventual circulation of international clones, as revealed by two and seven isolates of CC235 and CC244, respectively, previously detected in the five continents.

Antimicrobial resistance decreased in more recent isolates, with a statistically significant difference for amikacin. Data for isolates of the present study were additionally analysed according to the same breakpoints used for isolates of Pellegrino et al. (2002); the difference increased for carbapenems and piperacillin-tazobactam. These results are consistent with the decline of SPM-positive isolates and the absence of the $r m t D$ gene in the more recent isolates.

The mechanism of carbapenem resistance was thoroughly characterised for a subset of isolates, including all obtained from infections. Carbapenemase production was absent, and resistant isolates displayed the classic carbapenem resistance determinants. Inactivating mutations in $\operatorname{oprD}$ gene described in previous studies (Xavier et al. 2010, Castanheira et al. 2014) were fully congruent with imipenem resistance and associated with higher MIC values when AmpC overproduction was present. Efflux pump overproduction was constantly detected in association with other carbapenem-resistance mechanisms. All three isolates exhibiting AmpC overproduction as the single resistance mechanism were $\beta$-lactam susceptible. In fact, AmpC overproduction is a gradual and complex phenomenon, and the occurrence of $\beta$-lactam resistance is unpredictable (Lister et al. 2009). This observation of the present study indicates the assay used had sufficient sensitivity, and the isolates did not have a wild-type AmpC-producing phenotype. The clinical implication of this result remains a challenge (Lister et al. 2009). The finding of carbapenem-susceptible isolates with efflux overexpression or $\mathrm{AmpC}$ overproduction reflects the notion that resistance in $P$. aeruginosa is mostly determined by multiple mechanisms, each with slightly decreasing susceptibility, depending on each strains' genetic background (Castanheira et al. 2014).

We observed that the SPM-1 clone decreased in frequency when isolates obtained in two timeframes eight year apart were compared. This exchange could have been driven by the emergence of carbapenem-resistant Acinetobacter baumannii international clones in the study hospital, as previously reported (Martins et al. 2013), and of other resistant isolates, including P. aeruginosa. The study isolates were collected from 2007 to 
TABLE V

Characteristics of sequence-types (ST) detected among clinical and environmental study isolates

\begin{tabular}{|c|c|c|c|c|}
\hline Isolate & Antimicrobial resistance Profile & ST & $\mathrm{CC}$ & Countries \\
\hline \multicolumn{5}{|c|}{ Clinical Isolates } \\
\hline PCI032 & $\begin{array}{l}\text { AMK, FEP, CIP, CAZ, } \\
\text { TZP, TOB, GEN }\end{array}$ & 1844 & Singleton & Brazil \\
\hline PCI042 & $\begin{array}{l}\text { MEM, FEP, CIP, IPM, } \\
\text { CAZ, TZP, TOB, GEN }\end{array}$ & 244 & 244 & $\begin{array}{c}\text { Australia, Central African Republic, } \\
\text { China, France, Ivory Coast, Nigeria, } \\
\text { Poland, Russia, Spain, UK }\end{array}$ \\
\hline PCI045 & Susceptible & 235 & 235 & Australia, Belarus, Brazil, \\
\hline PCI124 & $\begin{array}{l}\text { MEM, AMK, FEP, CIP, } \\
\text { IPM, TZP, TOB, GEN }\end{array}$ & & & $\begin{array}{l}\text { Central African Republic, China, } \\
\text { Croatia, France, Hungary, } \\
\text { Ivory Coast, Kazakhstan, Nigeria, } \\
\text { Norway, Poland, Republic of Belarus, } \\
\text { Russia, Serbia, Singapore, Spain }\end{array}$ \\
\hline PCI059 & IPM & 890 & Singleton & Australia, Brazil \\
\hline PCI073 & Susceptible & 1767 & 244 & Brazil \\
\hline PCI126 & Susceptible & 532 & 244 & Australia, Brazil, France, Spain \\
\hline PCI133 & Susceptible & 1027 & SLV of 2223 & Brazil \\
\hline PCC457 & $\begin{array}{c}\text { MEM, FEP, CIP, IPM, CAZ, } \\
\text { TZP, TOB, GEN }\end{array}$ & 1768 & 244 & Brazil \\
\hline PCC524 & FEP & 1769 & 195 & Brazil \\
\hline PCC762 & $\begin{array}{l}\text { MEM, AMK, FEP, CIP, } \\
\text { IPM, CAZ, TOB, GEN }\end{array}$ & 1602 & SLV of ST1937 & Brazil \\
\hline
\end{tabular}

Environmental isolates

\begin{tabular}{lcccc}
\hline CTI9.2B & AMK, CAZ, FEP, GEN, & 1602 & SLV of \\
IPM, CIP, TOB & & ST1937 & Brazil \\
CTI9.2A & AMK, CAZ, FEP, GEN, & 1603 & 244 & Brazil \\
CTI19 & IPM, CIP, TOB & & & Australia, Brazil, France, Spain \\
CTI33 & Susceptible & 446 & 244 & Australia, Austria, Brazil, China, \\
& CAZ, FEP, GEN, IPM, & 277 & 244 & Central African Republic, Spain \\
\hline
\end{tabular}

AMK: amikacin; CAZ: ceftazidime; CIP: ciprofloxacin; FEP: cefepime; GEN: gentamicin; IPM: imipenem; MEM: meropenem; TZP: piperacillin-tazobactam; TOB: tobramycin; SLV: single-locus variant.

2008, but we believe the SPM-1 clone was rare in the hospital until recently. A review of the surveillance data for multidrug-resistant pathogens from August 2013 to July 2014 revealed only two isolates with a colistin-only susceptible profile, suggestive of SPM-1 producing strains.

SPM-1 $P$. aeruginosa fading could also be explained by a decrease in bacterial fitness, as proposed by others to occur with highly antimicrobial resistant strains (Andersson \& Hughes 2010). A better understanding of the mechanisms of emergency, spread and dissemination of pandemic clones is needed. As antimicrobial resistance becomes a global public health threat, this knowledge will be essential for the development of modern efficient strategies to overcome infections.

\section{REFERENCES}

Andersson DI, Hughes D. Antibiotic resistance and its cost: is it possible to reverse resistance? Nat Rev Microbiol. 2010; 8(4): 260-71.
Bizzini A, Durussel C, Bille J, Greub G, Prod'hom G. Performance of matrix-assisted laser desorption ionization-time of flight mass spectrometry for identification of bacterial strains routinely isolated in a clinical microbiology laboratory. J Clin Microbiol. 2010; 48(5): 1549-54.

Castanheira M, Deshpande LM, Costello A, Davies TA, Jones RN. Epidemiology and carbapenem resistance mechanisms of carbapenem-non-susceptible Pseudomonas aeruginosa collected during 2009-11 in 14 European and Mediterranean countries. J Antimicrob Chemother. 2014; 69(7): 1804-14.

CLSI - Clinical and Laboratory Standards Institute. Performance standards for antimicrobial susceptibility testing: twenty-third informational supplement M100-S23. Wayne: CLSI; 2013. 199 pp.

Cornaglia G, Giamarellou H, Rossolini GM. Metallo- $\beta$-lactamases: a last frontier for $\beta$-lactams? Lancet Infect Dis. 2011; 11(5): 381-93.

Corrêa LL, Montezzi LF, Bonelli RR, Moreira BM, Picão RC. Revised and updated multiplex PCR targeting acquired 16S rRNA methyltransferases. Int J Antimicrob Agents. 2014; 43(5): 479-81. 
Curran B, Jonas D, Grundmann H, Pitt T, Dowson CG. Development of a multilocus sequence typing scheme for the opportunistic pathogen Pseudomonas aeruginosa. J Clin Microbiol. 2004; 42(12): 5644-9.

Doi Y, Garcia D, Adams J, Paterson DL. Coproduction of novel 16S rRNA methylase RmtD and metallo-b-lactamase SPM-1 in a panresistant Pseudomonas aeruginosa isolate from Brazil. Antimicrob Agents Chemother. 2007; 51(3): 852-6.

Fontes LC, Neves PR, Oliveira S, Silva KC, Hachich EM, Sato MIZ, et al. Isolation of Pseudomonas aeruginosa coproducing metallob-lactamase SPM-1 and 16S rRNA methylase RmtD1 in an urban river. Antimicrob Agents Chemother. 2011; 55(6): 3063-4.

Francisco AP, Vaz C, Monteiro PT, Melo-Cristino J, Ramirez M, Carriço JA. PHYLOViZ: phylogenetic inference and data visualization for sequence based typing methods. BMC Bioinformatics. 2012; 13: 87.

Hall TA. BioEdit: a user-friendly biological sequence alignment editor and analysis program for Windows 95/98/NT. Nucleic Acids Symp Ser. 1999; 41: 95-8.

Horan TC, Andrus M, Dudeck MA. CDC/NHSN surveillance definition of health care-associated infection and criteria for specific types of infections in the acute care setting. Am J Infect Control. 2008; 36(5): 309-32.

Hossain A, Ferraro MJ, Pino RM, Dew RB, Moland ES, Lockhart TJ, et al. Plasmid-mediated carbapenem-hydrolyzing enzyme KPC2 in an Enterobacter sp. Antimicrob Agents Chemother. 2004; 48(11): 4438-40.

Lister PD, Wolter DJ, Hanson ND. Antibacterial-resistant Pseudomonas aeruginosa: clinical impact and complex regulation of chromosomally encoded resistance mechanisms. Clin Microbiol Rev. 2009; 22(4): 582-610.

Lomovskaya O, Warren MS, Lee A, Galazzo J, Fronko R, Lee M, et al. Identification and characterization of inhibitors of multidrug resistance efflux pumps in Pseudomonas aeruginosa: novel agents for combination therapy. Antimicrob Agents Chemother. 2001; 45(1): 105-16.

Maatallah M, Cheriaa J, Backhrouf A, Iversen A, Grundmann H, Do T, et al. Population structure of Pseudomonas aeruginosa from five mediterranean countries: evidence for frequent recombination and epidemic occurrence of CC235 PLoS ONE. 2011; 6(10): e25617.

Mahenthiralingam E, Campbell ME, Foster J, Lam JS, Speert DP. Random amplified polymorphic DNA typing of Pseudomonas aeruginosa isolates recovered from patients with cystic fibrosis. J Clin Microbiol. 1996; 34(5): 1129-35.

Martins N, Dalla-Costa L, Uehara AA, Riley LW, Moreira BM. Emergence of Acinetobacter baumannii international clone II in Brazil: reflection of a global expansion. Infect Genet Evol. 2013; 20: 378-80.

Mendes RE, Kiyota KA, Monteiro J, Castanheira M, Andrade SS, Gales AC, et al. Rapid detection and identification of metallo-b- lactamase-encoding genes by multiplex real-time PCR assay and melt curve analysis. J Clin Microbiol. 2007; 45(2): 544-7.

Nordmann P, Poirel L, Dortet L. Rapid detection of carbapenemase-producing Enterobacteriaceae. Emerg Infect Dis. 2012; 18(9): 1503-7.

Papp-Wallace KM, Endimiani A, Taracila MA, Bonomo RA. Carbapenems: past, present, and future. Antimicrob Agents Chemother. 2011; 55(11): 4943-60.

Pellegrino FLPC, Casali N, Nouér SA, Riley LW, Moreira BM. A carbapenem-susceptible Pseudomonas aeruginosa strain carrying the bla $_{\mathrm{SPM}}$ gene. Diagn Microbiol Infect Dis. 2008; 61(2): 214-6.

Pellegrino FLPC, Teixeira LM, Carvalho MG, Nouer SA, de Oliveira MP, Sampaio JLM, et al. Occurrence of a multidrug-resistant Pseudomonas aeruginosa clone in different hospitals in Rio de Janeiro, Brazil. J Clin Microbiol. 2002; 40(7): 2420-4.

Picão RC, Andrade SS, Nicoletti AG, Campana EH, Moraes GC, Mendes RE, et al. Metallo-b-lactamase detection: comparative evaluation of double-disk synergy versus combined disk tests for IMP-, GIM-, SIM-, SPM-, or VIM-producing isolates. J Clin Microbiol. 2008; 46(6): 2028-7.

Picão RC, Carrara-Marroni FE, Gales AC, Venâncio EJ, Xavier DE, Tognim MCB, et al. Metallo- $\beta$-lactamase-production in meropenem-susceptible Pseudomonas aeruginosa isolates: risk for silent spread. Mem Inst Oswaldo Cruz. 2012; 107(6): 747-51.

Poirel L, Le Thomas I, Naas T, Karim A, Nordmann P. Biochemical sequence analyses of GES-1, a novel class A extended-spectrum $\beta$-lactamase, and the class 1 integron In52 from Klebsiella pneumoniae. Antimicrob Agents Chemother. 2000; 44(3): 622-32.

Rodríguez-Martinez J-M, Poirel L, Nordmann P. Extended-spectrum cephalosporinases in Pseudomonas aeruginosa. Antimicrob Agents Chemother. 2009a; 53(5): 1766-71.

Rodríguez-Martinez J-M, Poirel L, Nordmann P. Molecular epidemiology and mechanisms of carbapenem resistance in Pseudomonas aeruginosa. Antimicrob Agents Chemother. 2009b; 53(11): 4783-8.

Silva FM, Carmo MS, Silbert S, Gales AC. SPM-1-producing Pseudomonas aeruginosa : analysis of the ancestor relationship using multilocus sequence typing, pulsed-field gel electrophoresis, and automated ribotyping. Microb Drug Resist. 2011; 17(2): 215-20.

Toleman MA. Molecular characterization of SPM-1, a novel metallobeta-lactamase isolated in Latin America: report from the SENTRY antimicrobial surveillance programme. J Antimicrob Chemother. 2002; 50(5): 673-9.

Xavier DE, Picão RC, Girardello R, Fehlberg LC, Gales AC. Efflux pumps expression and its association with porin down-regulation and $\beta$-lactamase production among Pseudomonas aeruginosa causing bloodstream infections in Brazil. BMC Microbiol. 2010; 10: 217. 